\title{
Hydrogen sulfide inhibits endoplasmic reticulum stress through the GRP78/mTOR pathway in rat chondrocytes subjected to oxidative stress
}

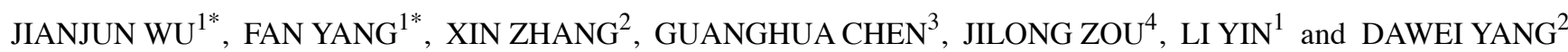 \\ ${ }^{1}$ Department of Cardiology, The Second Affiliated Hospital of Harbin Medical University, Harbin, Heilongjiang 150086; \\ ${ }^{2}$ Department of Orthopedic Surgery, The Fourth Affiliated Hospital of Harbin Medical University, Harbin, \\ Heilongjiang 150001; ${ }^{3}$ Department of Orthopedic Surgery, The Second Affiliated Hospital of Harbin Medical University; \\ ${ }^{4}$ Department of Orthopedic Surgery, The First Affiliated Hospital of Harbin Medical University, \\ Harbin, Heilongjiang 150086, P.R. China
}

Received May 29, 2020; Accepted December 29, 2020

DOI: $10.3892 / \mathrm{ijmm} .2021 .4867$

\begin{abstract}
The activation of oxidative stress is a primary cause of chondrocyte apoptosis in osteoarthritis (OA). The 78-kDa glucose-regulated protein (GRP78)/mammalian target of rapamycin (mTOR) signaling pathway has been demonstrated to be linked with the endoplasmic reticulum (ER) and autophagy. Hydrogen sulfide $\left(\mathrm{H}_{2} \mathrm{~S}\right)$ has been reported to exert antioxidant effects. The present study investigated oxidative stress levels via 2',7'-dichlorofluorescin diacetate and MitoSOX staining, apoptosis rates via flow cytometry and the expression levels of ER stress-related proteins in GYY4137 (donor of $\mathrm{H}_{2} \mathrm{~S}$ )-treated chondrocytes (CHs). CHs were isolated from the bilateral hip joints of male rats to examine mitochondrial permeability transition pore opening- and mTOR signaling pathway-related proteins. The results demonstrated that tert-Butyl hydroperoxide (TBHP) increased $\mathrm{CH}$ apoptosis, and treatment with GYY4137 ameliorated TBHP-mediated the generation of ROS and $\mathrm{CH}$ apoptosis. Moreover, TBHP-treated $\mathrm{CHs}$ displayed elevated ER stress sensor expression levels and apoptotic rates; however, the TBHP-induced protein expression levels were decreased following GYY4137 treatment. In the present study, treatment with either GYY4137 or transfection with GRP78
\end{abstract}

Correspondence to: Professor Dawei Yang, Department of Orthopedic Surgery, The Fourth Affiliated Hospital of Harbin Medical University, 37 Yiyuan Road, Nangang, Harbin, Heilongjiang 150001, P.R. China

E-mail: yangdawei9797@sina.com

*Contributed equally

Abbreviations: OA, osteoarthritis; ROS, reactive oxygen species; NAC, $N$-acetyl-L-cysteine; ER, endoplasmic reticulum; $\mathrm{H}_{2} \mathrm{~S}$, hydrogen sulfide; SOD, superoxide dismutase

Key words: osteoarthritis, oxidative stress, chondrocytes, endoplasmic reticulum stress, hydrogen sulfide
siRNA both suppressed the activation of p-P70S6k and p-mTOR. $\mathrm{H}_{2} \mathrm{~S}$ played an important role in regulating ER stress in TBHP-stimulated CHs. GYY4137 promoted autophagy, which was accompanied by the inhibition of ER stress. On the whole, the present study demonstrates that TBHP-induced oxidative stress stimulates ER interactions and $\mathrm{CH}$ apoptosis, which are suppressed by exogenous $\mathrm{H}_{2} \mathrm{~S}$ via modulating the GRP78/mTOR signaling pathway.

\section{Introduction}

Osteoarthritis (OA) is a progressive disease, characterized by cartilage erosion, osteophyte formation, chronic low-grade inflammation of the synovium and abnormal bone remodeling. Due to advances being made in analgesia and in arthroplasty, the survival rate of patients with OA has improved, and their quality of life has significantly improved. OA is not traditionally regarded as an inflammatory disease, although a number of studies have confirmed that oxidative stress affects the pathogenesis of OA (1). Oxidative stress is closely associated with vascular inflammation as reactive oxygen species (ROS) overproduction creates a pro-inflammatory microenvironment and elevates inflammatory cytokine levels. The release of inflammatory cytokines into the synovial fluid stimulates the production of pro-inflammatory cytokines, which could contribute to the pathology of OA $(2,3)$.

Chondrocytes ( $\mathrm{CHs}$ ), the only cell type present in cartilage, are responsible for the development of cartilage $(4,5)$. $\mathrm{CHs}$ produce and maintain the extracellular matrix, which normally has a low turnover rate. The widespread use of non-steroidal anti-inflammatory drugs is accompanied by an increase in gastrointestinal reactions, which affects the survival of patients. To overcome bioavailability and stability issues, intra-articular injections with small molecules are rapidly cleared from the joint within hours via synovial vasculature and larger macromolecules within days via synovial lymphatics (5-7). However, drugs for OA have limited success and display challenges with obtaining effective drug loading and release profiles persist $(8,9)$. Therefore, attenuating the 
deleterious effects of oxidative stress on $\mathrm{CHs}$ may serve as a strategy with which to repair cartilage tissue, In particular, their application to oxidative stress mechanisms requires further investigation.

Increased ROS generation and unfolded protein responses (UPRs) in the $\mathrm{CH}$ microenvironment can activate endoplasmic reticulum (ER) stress, and mitochondrial injury is associated with rat $\mathrm{CH}$ apoptosis (10-12). ER stress in $\mathrm{CHs}$ plays an important role in the pathogenesis of OA. Studies have revealed that cells respond to ER stress by activating the UPR, a signaling network consisting of three primary molecular sensors, including eukaryotic translation initiation factor $2 \alpha$ kinase 3, activating transcription factor-6 (ATF6) and inositol-requiring enzyme $1 \alpha(13-15)$. Under physiological conditions, these proteins are associated with Bip or ER chaperone protein glucose-regulated protein 78 (GRP78) and are engaged in inactivation. Previous research has revealed that the transcriptional activity of GRP78 is significantly elevated up to 10-25 fold during suffering from the stress response (16). There are 3 classical branches of the signaling pathway: PERK, ATF6 and IRE do not operate independently. They constitute an intricate signaling network and crosstalk dynamics decide cell fate with respect to survival or death following ER stress. All 3 pathways transduce information to repair the ER status and restore protein-folding capacity in ROS-exposed CHs. C EBP-homologous protein (CHOP) gadd153 (or simply CHOP) is a transcription factor which is the most important in regulating the ER stress-dependent apoptosis. In CHs in cartilage, ER stress can increase the expression of CHOP (16); thus, inhibiting ER stress may serve as a novel therapeutic approach for OA.

Hydrogen sulfide $\left(\mathrm{H}_{2} \mathrm{~S}\right)$ has been proposed as a potential regulator of inflammation and is a novel signaling molecule with potent cytoprotective actions. Moreover, $\mathrm{H}_{2} \mathrm{~S}$ is an endogenously produced gasotransmitter that has been reported to be involved in the inhibition of oxidative stress (17). The physiological and pathophysiological regulation of $\mathrm{H}_{2} \mathrm{~S}$ in cellular function has received increasing attention. Compared with patients with rheumatoid arthritis, the concentrations of $\mathrm{H}_{2} \mathrm{~S}$ in synovial fluid aspirates and plasma samples have been shown to be 2-fold higher in paired patients with non-inflammatory arthritis (18). GYY4137 has been well-known as a novel $\mathrm{H}_{2} \mathrm{~S}$ donor with its multiple bio- activities (19-21). The present study examined the effects of exogenous $\mathrm{H}_{2} \mathrm{~S}$ on tert-Butyl hydroperoxide (TBHP)-induced ER stress mitochondria-associated apoptotic signaling pathways in CHs, as well as the underlying mechanisms.

\section{Materials and methods}

Animals. A total of 40 male SD rats (weighing, 250-280 g; age, 8 weeks) were obtained from the experimental animal center of Harbin Medical University, and kept at the Key Laboratory Of Molecular Imaging (Harbin, China). Rats were acclimated for 7 days prior to the surgeries and were provided with unrestricted access to food and water at room temperature $\left(22-26^{\circ} \mathrm{C}\right)$ at $10-30 \%$ humidity. Animals were performed according to guidelines approved by the Institutional Experimental Animal Care and Ethics Committee of Harbin Medical University (Harbin, China) and with approval from the Harbin Medical University Ethics Review Board.
Cell isolation and culture. All animal care and experimental protocols complied with the Guide for the Care and Use of Laboratory Animals of Harbin Medical University (Harbin, China). Rats were anesthetized with chloral hydrate $(3.5 \%$, $350 \mathrm{mg} / \mathrm{kg}$, i.p.). The bilateral hip joints of male rats were isolated immediately and washed thoroughly with normal saline and then immediately processed for biochemical and morphological analyses. Each bilateral hip joint was subjected to the following analyses: Cell viability, western blot analysis for ER stress pathway-associated protein indexes, ROS activity, fluorescence staining and flow cytometric analyses. Primary $\mathrm{CHs}$ were isolated from the bilateral hip joints of the male rats using $0.25 \%$ trypsin for digestion lasting approximately $1 \mathrm{~h}$ at $37^{\circ} \mathrm{C}$ and incubated with collagenase II $(1.5 \mathrm{mg} / \mathrm{ml})$ in DMEM at $37^{\circ} \mathrm{C}$ and $5 \% \mathrm{CO}_{2}$ for $6 \mathrm{~h}$. The $\mathrm{CH}$ suspensions were then centrifuged at $250 \times \mathrm{g} 4^{\circ} \mathrm{C}$ for $5 \mathrm{~min}$ and cultured at $37^{\circ} \mathrm{C}$ and $5 \% \mathrm{CO}_{2}$ in DMEM complete culture medium (10\% FBS, $100 \mu \mathrm{g} / \mathrm{ml}$ streptomycin and $100 \mathrm{U} / \mathrm{ml}$ penicillin). To avoid the loss of phenotype, rat $\mathrm{CHs}$ were not used for $>2$ passages in the in vitro experiments. At the end of the experimental procedure, the animals were sacrificed by an i.p. injection of an overdose of sodium pentobarbital $(100 \mathrm{mg} / \mathrm{kg})$.

Reagents. GYY4137 (SML0100; chemical structure shown in Fig. 1A), tert-Butyl hydroperoxide solution (416665), $\mathrm{N}$-acetylcysteine (NAC, A9165), chloroquine (CQ, C6628) were obtained from Sigma-Aldrich; Merck KGaA. DMEM/F12 (SH30023.01), FBS and type II collagenases were purchased from HyClone; Cytiva. 2,7-Dichlorofluorescein diacetate (DCFH-DA) was purchased from Beyotime Institute of Biotechnology. Lipofectamine 2000, calcium green-5N and ER-tracker were both obtained from Invitrogen; Thermo Fisher Scientific, Inc. Primary antibodies [GRP78, \#3177; ATF6, \#65880; CHOP, \#2895; phospho-p70S6 kinase, \#9208; p70S6 kinase, \#2708; mammalian target of rapamycin (mTOR), \#2983; phospho-mTOR, \#5536; SQSTM1/p62, \#23214; LC3A/B, \#4108] were provided by Cell Signaling Technology, Inc. and secondary antibodies (\#SA00001-1 and \#SA00001-2) were purchased from ProteinTech Group, Inc., respectively.

Cell viability assay. Cell counting kit-8 (CCK-8) assay was performed for the assessment of the cytotoxicity of GYY4137 (100 $\mu \mathrm{M}, 0-24 \mathrm{~h})$ and TBHP $(0-50 \mu \mathrm{M}, 0-24 \mathrm{~h})$ on CHs. A total of $10 \mu \mathrm{l}$ CCK-8 solution was added to each 96 -well plates $\left(5 \times 10^{3}\right.$ cells per well) and co-incubated at $37^{\circ} \mathrm{C}$ for $4 \mathrm{~h}$. The absorbance was measured at $450 \mathrm{~nm}$ using a microplate reader (InfiniteM200, Tecan Group, Ltd.).

Calculation of half maximal effective concentration $\left(E C_{50}\right)$. GraphPad Prism 7.0 software was used for the calculation of the $\mathrm{EC}_{50}$ of GYY4137 in vitro. The fitted dose-effect curve and $\mathrm{EC}_{50}$ value were generated and with automatic output following the inputting rate data of GYY4137 on chondrocytes and the setting of parameters on GraphPad Prism 7.0.

MTT assay. CHs $\left(5 \times 10^{3}\right.$ cells/well) were cultured in 96-well plates and exposed to $0.4 \mathrm{mg} / \mathrm{ml}$ MTT diluted in complete DMEM for $4 \mathrm{~h}$ at $37^{\circ} \mathrm{C}$. Cells were washed with PBS and 
A<smiles>COc1ccc(P(=S)([S-])N2CCOCC2)cc1</smiles>

D

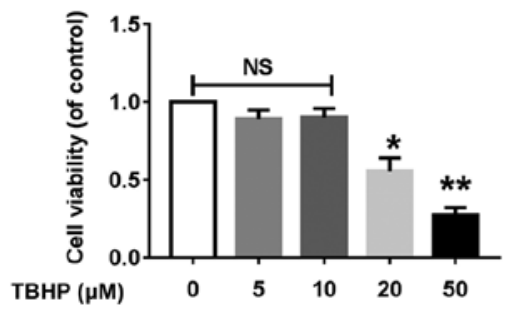

B

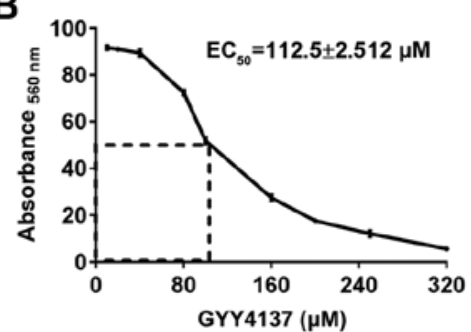

E

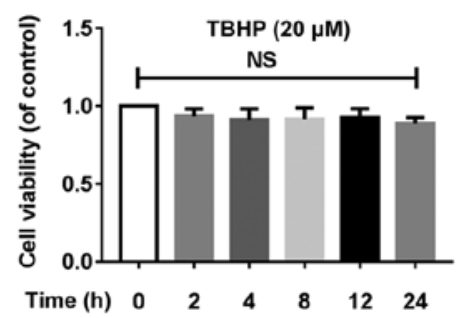

C

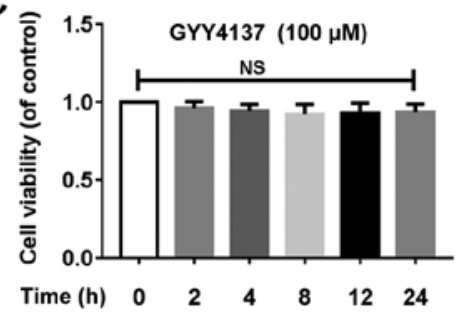

$\mathbf{F}$

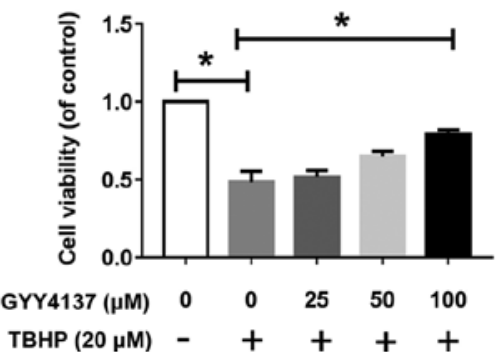

Figure 1. GYY4137 (100 $\mu \mathrm{M})$ reduces CH cell numbers without inducing apoptosis. (A) Chemical structure of GYY4137. (B) CH metabolic activity was analyzed by performing an MTT following treatment with GYY4137 dose range $\left(\mathrm{EC}_{50}, 112.5 \pm 2.5 \mu \mathrm{M}\right.$; $\left.\mathrm{n}=4\right)$. (C) Quantification of CHs viability following treatment with different time with GYY4137 treatment. (D) Quantification of CHs viability following treatment with increasing concentrations of TBHP (E) Quantification of CHs viability following treatment with different time with $20 \mu \mathrm{M}$ TBHP. (F) Quantification of CH viability following different dose of GYY4137 treatment following co-incubation with or without TBHP $20 \mu \mathrm{M}$. Data are presented as the means $\pm \mathrm{SEM}(\mathrm{n}=3)$. $(\mathrm{C}-\mathrm{F}){ }^{*} \mathrm{P}<0.05$ and ${ }^{* * *} \mathrm{P}<0.01$ vs. control. $\mathrm{CH}$, chondrocyte; TBHP, tert-Butyl hydroperoxide.

allowed to air-dry overnight. Formazan crystals were resuspended in DMSO and absorbance was measured at $560 \mathrm{~nm}$ with background at $670 \mathrm{~nm}$ on a Cytation 5 plate reader (BioTek Instruments, Inc.).

Observation of intracellular ROS content. The microscopic observation of intracellular ROS and mitochondrial ROS was performed by the oxidative conversion of cell permeable DCFH-DA to fluorescent 2', 7'-dichlorofluorescein (DCF) and MitoSOX (M36008, Invitrogen; Thermo Fisher Scientific, Inc.). CHs were divided into the control, TBHP, TBHP + GYY4137 and TBHP + NAC group, then stained with $10 \mu \mathrm{M}$ DCFH-DA solution or $5 \mu \mathrm{M}$ MitoSOX at $37^{\circ} \mathrm{C}$ for $20 \mathrm{~min}$ in the dark. The mean fluorescence intensity (MFI) of DCF and MitoSOX from 4 random fields was observed using an imaging system (BX50-FLA; Olympus Corporation) and analyzed using ImagePro Plus software (version 6.0, Media Cybernetics).

Measurement of superoxide dismutase (SOD) and catalase (CAT) activities. The Total Superoxide Dismutase Assay kit with NBT (Beyotime Institute of Biotechnology) and a Catalase Assay kit (Beyotime Institute of Biotechnology) were used to examine the activities of SOD and CAT as previously described (14). The activity results are expressed as U/mg protein.

Cell apoptosis analysis by flow cytometry. At least $10^{4}-10^{6}$ cells were analyzed by flow cytometry with the Annexin V/PI kit (BD Biosciences) using a Beckman Coulter MoFlo XDP. Briefly, the medium was removed and cells were treated with serum-reduced medium $(0.5 \%$ FBS $)$ with GYY4137 $(100 \mu \mathrm{M})$ or NAC $(5 \mu \mathrm{M})$ for $24 \mathrm{~h}$. Cells were suspended in $300 \mu \mathrm{l}$ binding buffer, and then stained with Annexin-FITC and propidium Iodide (PI). Positive controls for cell apoptosis or necrosis were indicated by staining with only Annexin-FITC or PI (Beckman Coulter, Inc.). Cell apoptosis was counted using summit V5.2.0.7477 software.

Calcium retention assay. Calcium retention capacities of rat $\mathrm{CH}$ mitochondria were measured as previously described (16) with some modifications. Briefly, primary $\mathrm{CHs}$ were incubated with $0.25 \mathrm{M}$ sucrose buffer for complex 1 without EGTA at $25^{\circ} \mathrm{C}$ (room temperature). Extra-mitochondrial calcium was visualized using a $500 \mathrm{~nm}$ excitation wavelength and emission wavelengths of $530 \mathrm{~nm}$ with $1 \mu \mathrm{M}$ calcium green $-5 \mathrm{~N}$. Experiments were performed in the presence and absence of $1.2 \mathrm{mM} \mathrm{MgCl}$ and $40 \mu \mathrm{M}$ ADP, as well as $1 \mathrm{mM}$ cyclosporine A (CsA), an inhibitor of the mitochondrial permeability transition pore (MPTP). The probe reversibly binds to calcium ions. The inclusion of CsA allows for an increased $\mathrm{Ca}^{2+}$ retention capacity prior to induction of the MPTP and the subsequent release of $\mathrm{Ca}^{2+}$ from the mitochondria.

Caspase activity assays. Caspase-3 activity was assessed using a colorimetric assay (ab39401, Abcam) according to the manufacturer's instructions. The activities of caspase-3, caspase- 8 and caspase- 9 were determined using a Multiplex Activity Assay kit (ab219915, Abcam) according to the manufacturer's instructions with some modifications. The protocol in the following link was followed: https:/www. abcam.com/ps/products/219/ab219915/documents/Caspase-3Caspase-8-and-Caspase-9-Multiplex-Activity-Assay-protocolbook-v1d-ab219915\%20(website).pdf. The caspase-3, -8 and -9 substrates were at room temperature prior to the experiment. All controls and samples were assayed in triplicate. The cells 
were plated overnight in DMEM at 2x10 $4 / 90 \mu$ l per well. Blank wells (DMEM without cells) contained $10 \mu 1$ of compound buffer. To assay caspase-3 activity in each well, assay loading solution was prepared by the addition of $50 \mu 1$ of caspase-3 substrate to $10 \mathrm{ml}$ of Assay Buffer. To assay tri-caspase activity in the same well, a mixed assay solution was prepared by the addition of $50 \mu \mathrm{l}$ of tri-caspase substrate to $10 \mathrm{ml}$ of assay buffer together. This was followed by the addition of $100 \mu \mathrm{l} /$ well of solution directly into cell plates without removing DMEM at room temperature for $45 \mathrm{~min}$. A microplate reader (InfiniteM200, Tecan Group Ltd.) was used to measure fluorescence at $\mathrm{Ex} / \mathrm{Em}=535 / 620 \mathrm{~nm}$ (caspase-3, red), $\mathrm{Ex} / \mathrm{Em}=490 / 525 \mathrm{~nm}$ (caspase-8, green), $\mathrm{Ex} / \mathrm{Em}=370 / 450 \mathrm{~nm}$ (caspase-8, blue).

GRP78 short interfering RNA transfection. GRP78 siRNA and scrambled siRNA (negative control) were designed and synthesized by Invitrogen; Thermo Fisher Scientific, Inc. (https://www.invivogen.com/sirnawizard/scrambled.php). The siRNA sequences were as follow: GRP78 target siRNA, 5'-GAA AGTATACCTCCAGTTTTT-3'; and scrambled siRNA, 5'-GTT CTAACGTCTATGACATTA-3'. siRNA targeting rat GRP78 was dissolved in siRNA suspension buffer and transfected into the $\mathrm{CHs}$ at a concentration of $150 \mathrm{nM}$; scrambled siRNA served as a negative control. All transient transfections were initially standardized to improve knockdown efficiency and subsequently performed with $150 \mathrm{nM}$ GRP78 siRNAs using Lipofectamine 2000 as per the manufacturer's protocol after $6 \mathrm{~h}$; transfection mixtures were replaced with regular medium. At $48 \mathrm{~h}$ following transfection, the cells were incubated with DMEM with or without TBHP for processing assessment of mitochondrial network and protein expression.

Western blot analysis. Total proteins from primary chondrocytes were extracted with RIPA buffer containing protease and phosphatase inhibitors (R0010, Beijing Solarbio Science $\&$ Technology Co., Ltd.) on ice. The concentration of proteins was measured by bicinchoninic acid (BCA) protein assay (Beyotime Institute of Biotechnology, Inc.). Equal amounts of protein $(50 \mu \mathrm{g})$ were separated by 10 or $12.5 \%$ SDS-PAGE (PG112/PG113, EpiZyme, inc.) and transferred onto $0.22 \mu \mathrm{M}$ NC membranes (P-N66485, Beijing Solarbio Science \& Technology Co., Ltd.). The membranes were blocked with $5 \%$ dried skimmed milk in Tris-buffered saline with $0.05 \%$ Tween-20 (TBS-T) for $1 \mathrm{~h}$ at room temperature with sustained shaking. The membrane was washed with TBS-T 3 times then probed with primary antibodies $(1: 1,000)$ by incubation at $4^{\circ} \mathrm{C}$ overnight. The following day, membranes were incubated with horseradish peroxidase (HRP)-conjugated secondary antibodies $(1: 5,000)$ for $2 \mathrm{~h}$ at room temperature. Immunoreactivity was visualized using ECL reagent (Wanlei Biotechnology) using ChemiDoc ${ }^{\mathrm{TM}}$ MP Imaging System (Tanon Science \& Technology Co., Ltd.). The densities of the bands were quantified using a Bio-Rad Chemi EQ densitometer and Quantity One software (Tanon Science \& Technology Co., Ltd.).

Staining procedures and microscopic analyses. For ER staining experiments, $0.5 \mu \mathrm{l}$ of ER-tracker green DMSO stock solution $(1 \mathrm{mM})$ was added into $2 \mathrm{ml}$ of pre-warmed DMEM incubated at $37^{\circ} \mathrm{C}, 5 \% \mathrm{CO}_{2}$ at room temperature for $30 \mathrm{~min}$ and washed with PBS solution 3 times. For Hoechst 33342/PI (CA1120, Beijing Solarbio Science \& Technology Co., Ltd.) dual-staining, CHs were firstly stained with Hoechst 33342 $(10 \mu \mathrm{M})$ for $30 \mathrm{~min}$ at $37^{\circ} \mathrm{C}$ and then stained with PI $(10 \mu \mathrm{M})$ for $5 \mathrm{~min}$. Cells were observed under fluorescence microscope (DMI4000B, Leica Microsystems, Inc.) after washing with PBS.

Statistical analysis. Data are presented as the means \pm the standard error of the mean (SEM) from at least 3 independent experiment replicates. One-way ANOVA followed by a Holm-Sidak test for post-hoc analysis using Graph Pad Prism 7. A value of $\mathrm{P}<0.05$ was considered to indicate a statistically significant difference.

\section{Results}

Effect of various concentrations of $\mathrm{H}_{2} \mathrm{~S}$ on $\mathrm{CH}$ viability with or without TBHP. The effect of GYY4137 on rat CHs viability was analyzed by performing an MTT assay. The $\mathrm{EC}_{50}$ (calculated using GraphPad Prism7) of GYY4137 in the CHs at $24 \mathrm{~h}$ was $112.5 \pm 2.5 \mu \mathrm{M}$ (Fig. 1B). Treatment with $100 \mu \mathrm{M}$ GYY4137 did not lead to any significant cytotoxicity from 0 to $24 \mathrm{~h}$, as shown by CCK-8 assay (Fig. 1C). Stimulation with TBHP at $20 \mu \mathrm{M}$ was selected to mimic oxidative stress associated with OA in the CHs, as TBHP has been reported to be more stable and long-lasting as an oxidative stress inducer compared with $\mathrm{H}_{2} \mathrm{O}_{2}$ (22). TBHP stimulation led to a significant decrease in cell viability in a time-dependent manner at the concentration of $20 \mu \mathrm{M}$ (Fig. 1D and E), as shown by CCK-8 assay. Moreover, GYY4137 reversed the decreased viability of CHs following co-incubation with TBHP $20 \mu \mathrm{M}$ (Fig. 1F). Collectively, these results indicated that treatment with $100 \mu \mathrm{M}$ GYY4137 reduced the total number of CHs by inhibiting cell proliferation, and cell apoptosis contributed more to the reduction in the total cell number of $\mathrm{CHs}$ at toxic GYY4137 concentrations ( $>200 \mu \mathrm{M})$. Therefore, GYY4137 at $100 \mu \mathrm{M}$ and TBHP at $20 \mu \mathrm{M}$ were selected for use in subsequent experiments.

GYY4137 protects CHs from oxidative stress-induced apoptosis. To determine whether treatment with an antioxidant could attenuate the apoptotic effects of TBHP, chondrocytes were treated with NAC. NAC is a derivative of L-cysteine that is well known for its ability to protects cells from ROS-induced cellular senescence. The results demonstrated that both cytoplasmic and mitochondrial ROS production were significantly increased in the TBHP group, an effect that was suppressed by GYY4137 or NAC treatment (Fig. 2A and B). Subsequently, SOD (Mn-SOD) and CAT (mito-CAT) activity in the mitochondria of the $\mathrm{CHs}$ was detected to determine the mechanisms through which GYY4137 suppresses ROS production. TBHP inhibited Mn-SOD and mito-CAT activity, and this was attenuated by GYY4137 or NAC treatment (Fig. 2C and D). GYY4137 markedly increased mitochondrial Mn-SOD and mito-CAT activity; therefore, it was hypothesized that exogenous $\mathrm{H}_{2} \mathrm{~S}$ can suppress mitochondrial ROS production. Flow cytometry was conducted to detect $\mathrm{CH}$ apoptosis (Fig. 3A, C and D). The proportion of early and late apoptotic cells (necrosis and dead cells) was markedly decreased following treatment with 
A
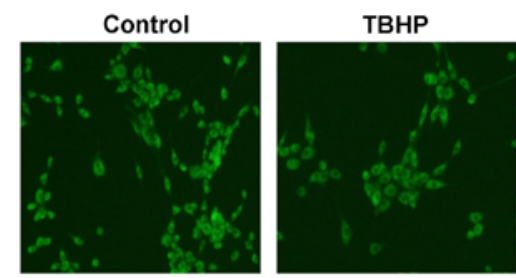

DCFH-DA

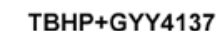

TBHP+NAC
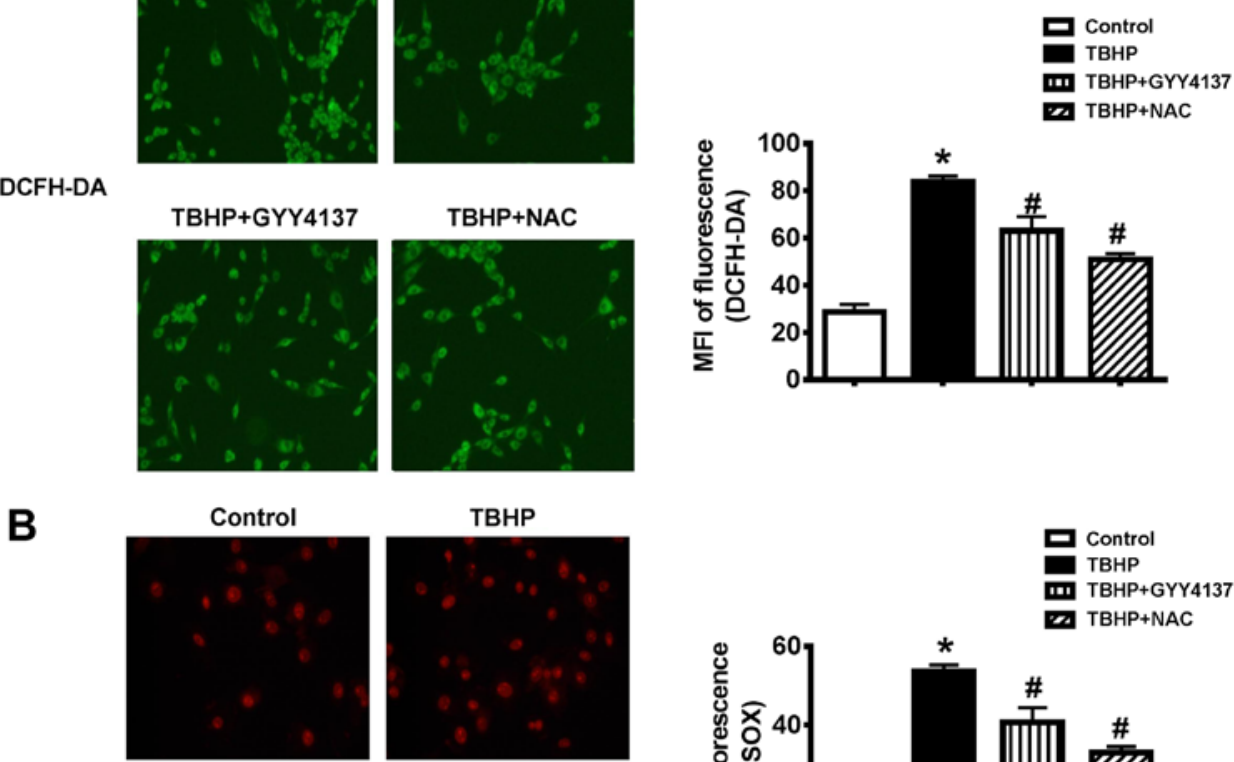

Mitosox

TBHP+GYY4137

TBHP+NAC
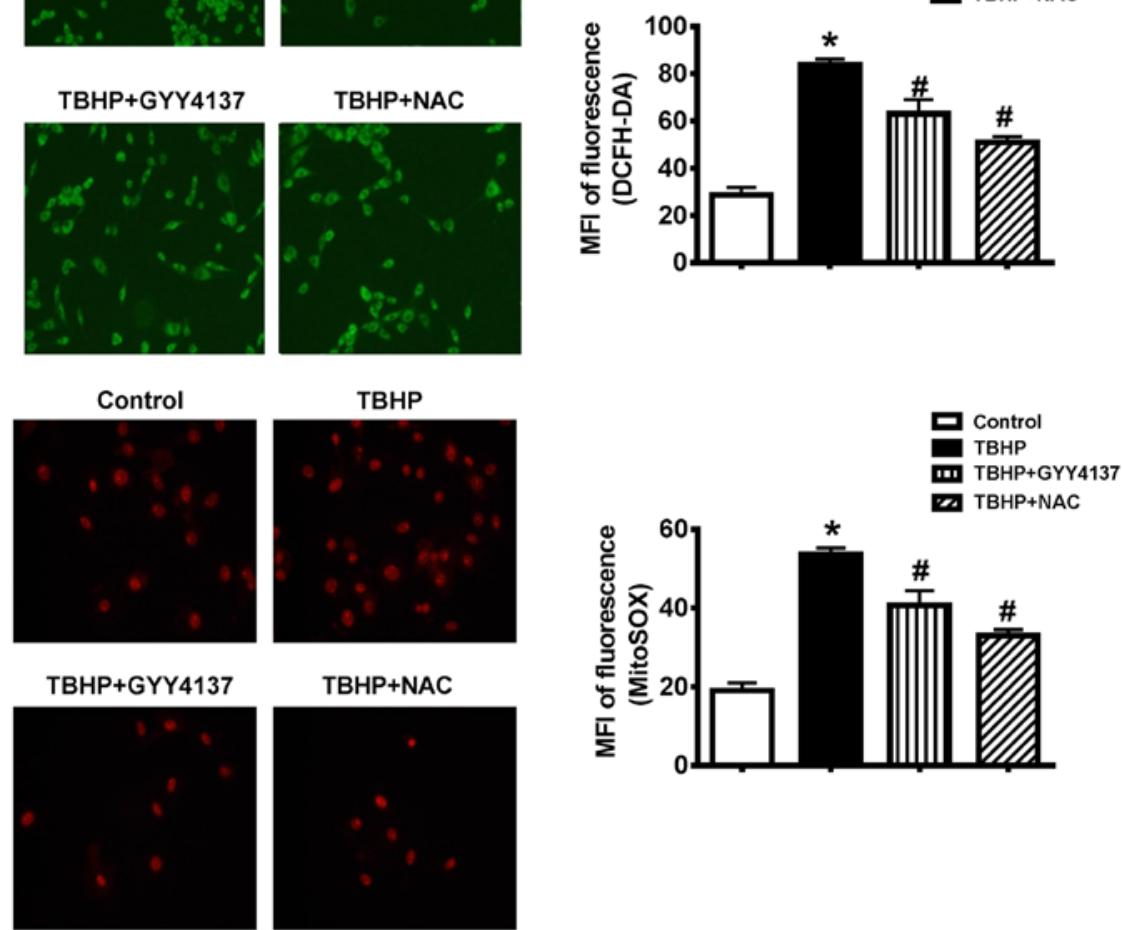

C

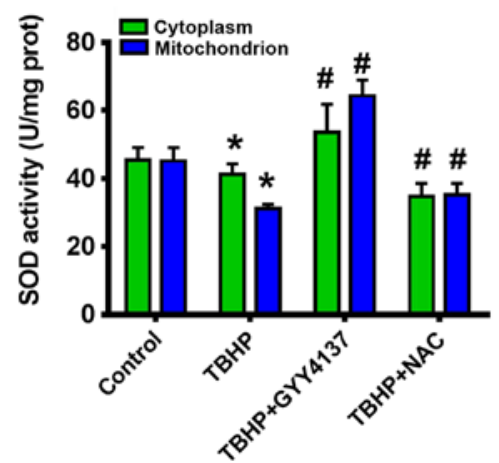

D

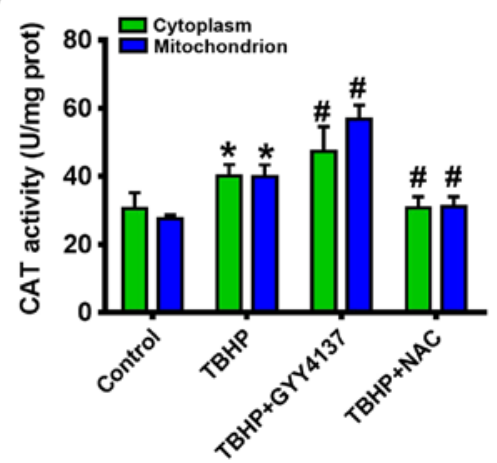

Figure 2. Effects of GYY4137 on hypoxia-induced overproduction of reactive oxygen species in the mitochondria of CHs. Random micrographs of (A) DCFH-DA and (B) MitoSOX-derived fluorescence in CHs. Quantitative analysis of the mean fluorescence intensity was obtained in the indicated groups. Magnification, x200. (C and D) Effects of GYY4137 on superoxide dismutase and catalase activity in CHs. Data are presented as the means \pm SEM ( $\mathrm{n}=3$ ). ${ }^{*} \mathrm{P}<0.05$ vs. control; " $\mathrm{P}<0.05$ vs. TBHP. CH, chondrocyte; DCFH-DA, 2',7'-dichlorofluorescin diacetate; TBHP, tert-Butyl hydroperoxide.

GYY4137 for $24 \mathrm{~h}$, which suggested that GYY4137 reversed TBHP-induced cell apoptosis (Fig. 3A, C and D).

Calcein $/ \mathrm{Co}^{2+}$ staining was conducted to examine whether calcium-dependent apoptosis induced by ER stress WAS mediated through the opening of $\mathrm{mPTP}$. The results revealed that mitochondrial calcein in the cytoplasm decreased gradually following TBHP stimulation and that GYY4137 or NAC treatment significantly inhibited mPTP opening at $24 \mathrm{~h}$, while treatment with NAC did not lead to any significant difference compared with GYY4137 treatment (Fig. 3B and E). The analysis of the activity of caspase-3 illustrated the anti-apoptotic effect of GYY4137 in the TBHP-stimulated CHs. Treatment with GYY4137 decreased caspase-3 activity compared with the TBHP group (Fig. 3F).
GYY4137 attenuates ER stress via the GRP78/mTOR signaling pathway. GRP78 is a key regulator of ER and previous research has reported that GRP78 overexpression aggravates ER stress (23). In the present study, siRNA against GRP78 decreased the GRP78 expression levels by $80 \%$ compared with the control group (Fig. 4A and B), which demonstrated the transfection efficiency of si-GRP78. Compared with the control group, DNA damage inducible transcript 3 (CHOP), GRP78 and ATF6 levels were significantly increased in the TBHP group, but decreased in the GYY4137 group (Fig. 4C and D). Moreover, the results suggested that ER stress occurs in $\mathrm{CHs}$, indicating the possibility of induction of a UPR-like event. ER-Tracker Green is highly selective for ER, consisting of the green-fluorescent dye and glibenclamide (24). Following 

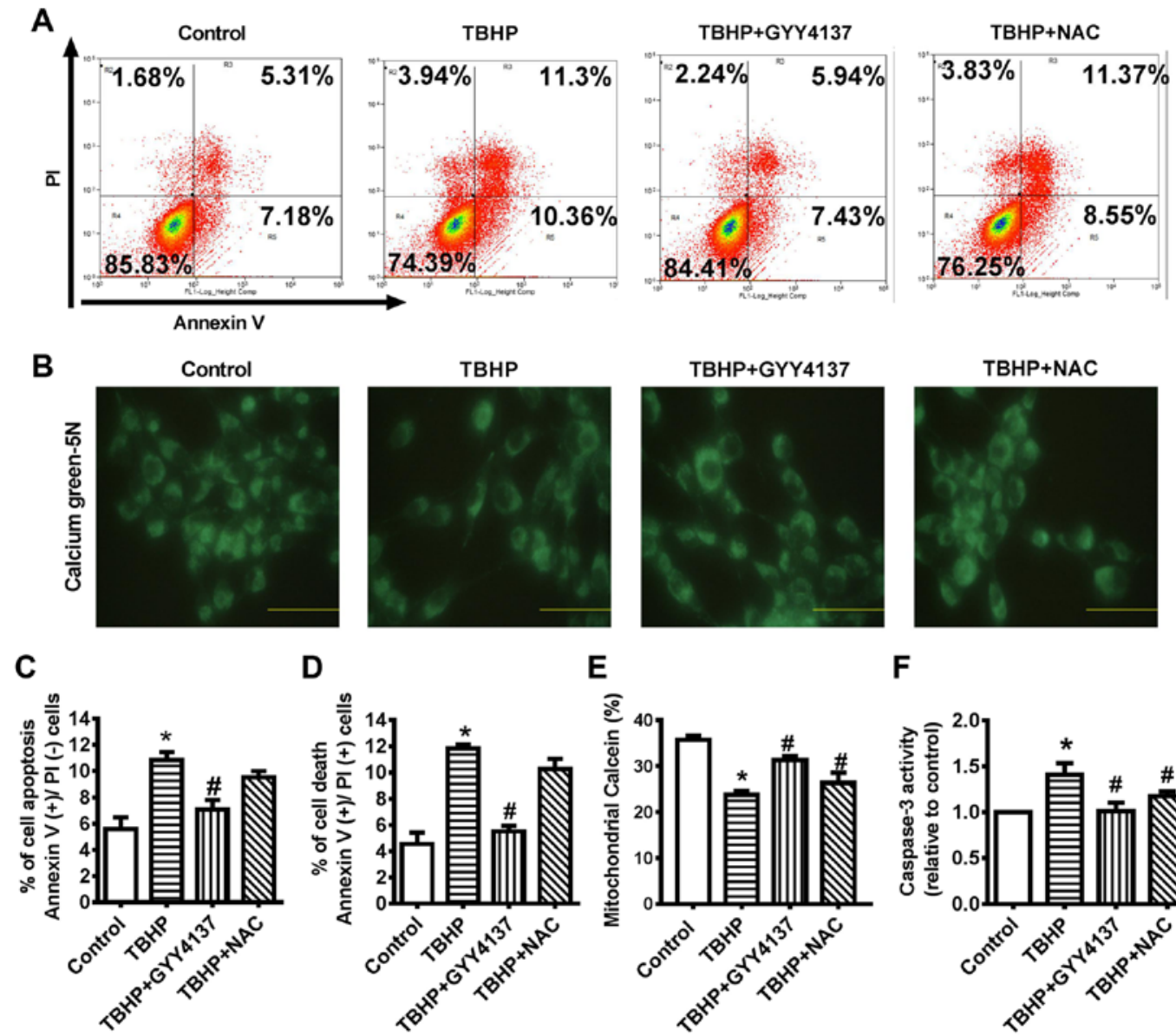

$\mathbf{F}$

Figure 3. GYY4137 protects CHs from oxidative stress-induced apoptosis. (A, C and D) Cells were treated with $0 \mu \mathrm{M}$ (control), $20 \mu \mathrm{M}$ TBHP, $100 \mu \mathrm{M}$ GYY4137 and $5 \mu \mathrm{M}$ NAC for $24 \mathrm{~h}$. Subsequently, cells were analyzed using a flow cytometer. Lower left quadrant, Annexin V/PI (live cells); upper left quadrant, Annexin $\mathrm{V}^{-} / \mathrm{PI}^{+}$(mechanically damaged cells); upper right quadrant, Annexin $\mathrm{V}^{+} / \mathrm{PI}^{+}$(late apoptotic or necrotic cells); lower right quadrant, Annexin $\mathrm{V}^{+} / \mathrm{PI}$ (early apoptotic cells). The experiments were repeated at least 3 times. ${ }^{*} \mathrm{P}<0.05$, significant difference vs. the control $(\mathrm{n}=4)$. (B and $\left.\mathrm{E}\right) \mathrm{CHs}$ were treated with $0 \mu \mathrm{M}$ (control), $20 \mu \mathrm{M}$ TBHP, $100 \mu \mathrm{M}$ GYY4137 and NAC for $24 \mathrm{~h}$. The mPTP opening was measured by staining with calcein- $\mathrm{AM}^{2}$ and COCl${ }_{2}(\mathrm{n}=5$ ). (F) Caspase-3 activity in CHs. Data are presented as the means \pm SEM. ${ }^{*} \mathrm{P}<0.05$ vs. control, ${ }^{~} \mathrm{P}<0.05$ vs. TBHP $(\mathrm{n}=3)$. TBHP, tert-Butyl hydroperoxide; NAC, $\mathrm{N}$-acetyl cysteine; $\mathrm{CH}$, chondrocyte; $\mathrm{mPTP}$, mitochondrial permeability transition pore.

transfection with si-GRP78, GYY4137 treatment protected the CHs against TBHP-induced ER stress, as measured by ER-tracker (Fig. 5A). Furthermore, the results demonstrated that the mean fluorescence intensity of ER-tracker was increased in the CHs in the TBHP-cultured group but was decreased following GYY4137 treatment for $24 \mathrm{~h}$ (Fig. 5B).

Chloroquine is an autophagy and Toll-like receptor (TLR) inhibitor, which is used to lock the late stages of autophagy in in vitro studies (chloroquine inhibits autophagic flux by decreasing autophagosome-lysosome fusion). Compared with the control group, the levels of p-P70S6K/P70S6K and $\mathrm{p}-\mathrm{mTOR} / \mathrm{mTOR}$ were significantly increased in the TBHP group. Compared with the TBHP group, the levels of p-P70S6K/P70S6K and p-mTOR/mTOR were significantly decreased following treatment with GYY4137, GRP78 siRNA or chloroquine. This suggested that GYY4137 suppressed the activation of the mTOR pathway induced by THBP through the inhibition of autophagy (Fig. 4E and F).

GRP78 knockdown leads to autophagy inhibition and cell dysfunction. The present study then determined whether GRP78 is necessary for increased ROS-induced cell apoptosis following TBHP stimulation by using siRNA to silence of GRP78 in CHs. The CHs were transfected with si-GRP78 to determine whether GRP78 was associated with GYY4137-induced autophagy. The results suggested that microtubule associated protein 1 light chain $3 \alpha$ (LC3) II/I expression levels were increased in the GYY4137 group compared with the TBHP group. Compared with the control group, the expression levels of $\mathrm{p} 62$ were decreased in the TBHP group, which suggested the suppression of autophagy (Fig. 4G and H). The CHs displayed an increased LC3II expression in the GYY4137 group compared with the TBHP group (Fig. 4G. There was no marked increase in autophagy following treatment with GRP78 siRNA compared with the control cells (Fig. 5A, LC3B). These results indicated that $\mathrm{H}_{2} \mathrm{~S}$ not only ameliorated autophagy, but also inhibited ER stress in a GRP78-dependent manner. Hoechst 33342/PI staining revealed that the ratio of apoptosis (Hoechst) and necrosis (PI) in the $\mathrm{CHs}$ was reduced $24 \mathrm{~h}$ following transfection with siRNA targeting GRP78 compared with the TBHP group (Fig. 5C). As shown in Fig. 5D, the CHs displayed a marked decrease in caspase activity folowing treatment with GYY4137. Moreover, the caspase activity of CHs decreased with GRP78 siRNA transfection, compared with the TBHP group. The results suggested that GRP78 activation was essential for 
A
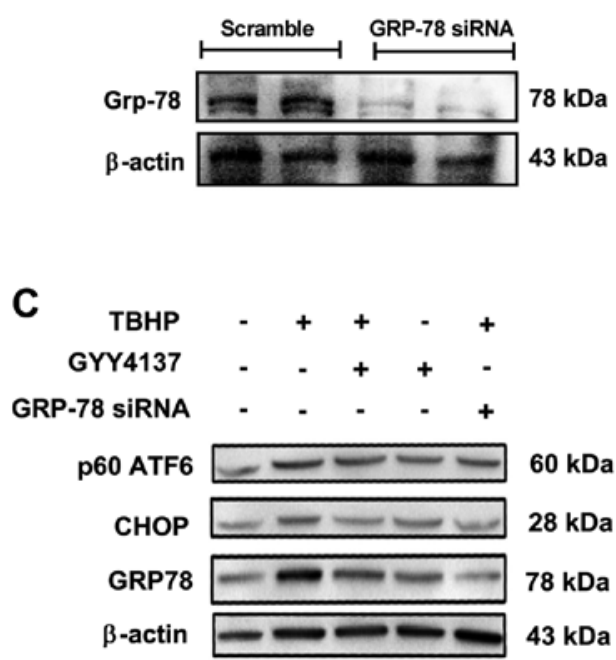

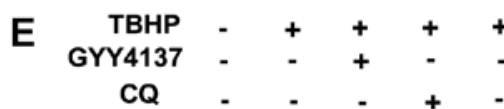

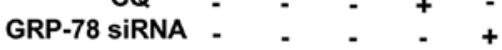

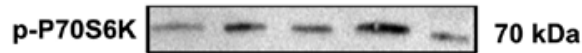

t-P70S6K

p-mTOR $=246 \mathrm{kDa}$

t-mTOR $=246$ kDa

$\beta$-actin $\longrightarrow-\square \mathrm{kDa}$
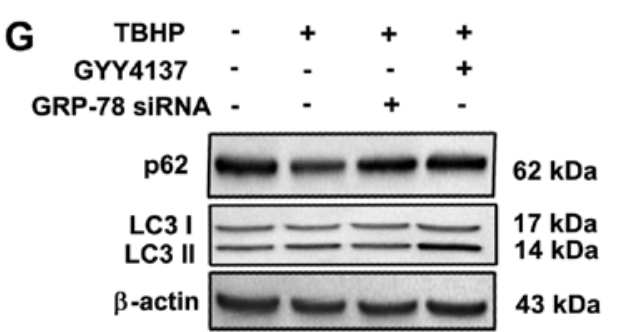
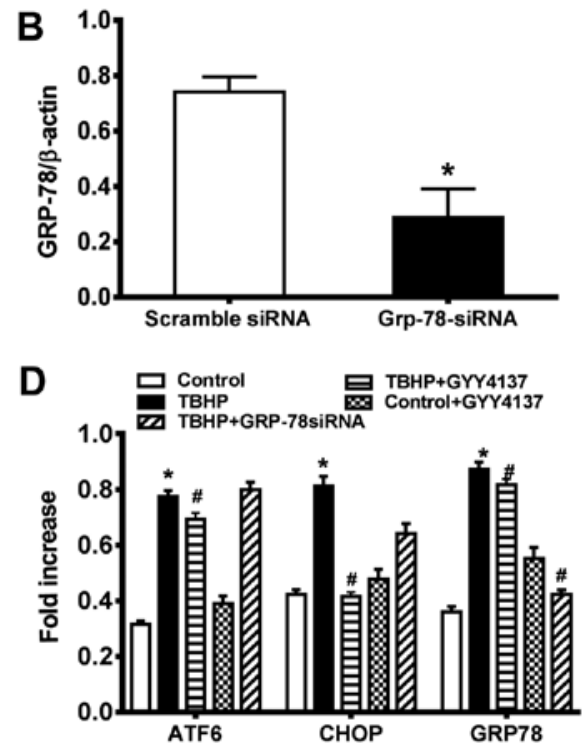

$\mathbf{F}$

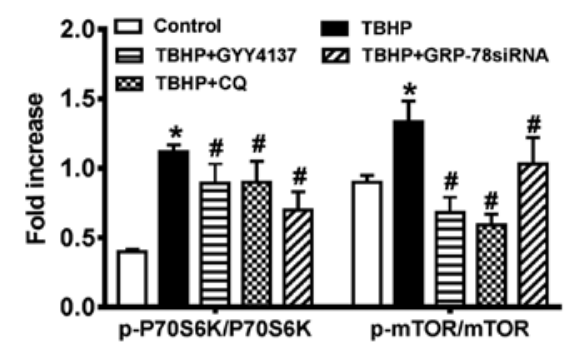

H

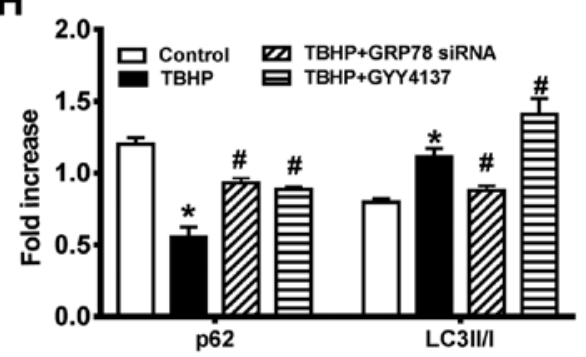

Figure 4. GRP78 knockdown leads to autophagy inhibition and cell dysfunction. (A) Protein expression levels were measured by western blot analysis. The following groups were assessed: i) Scramble siRNA; and ii) Grp-78 siRNA. (B) Densitometric results are expressed as a fold increase. (C) Protein expression levels were measured by western blot analysis. The following groups were assessed: i) Control, normal DMEM; ii) TBHP; iii) TBHP + GYY4137, CHs pre-treated with $100 \mu \mathrm{M}$ GYY4137; and iv) TBHP + GRP-78 siRNA, CHs transfected with $150 \mathrm{nM}$ GRP-78 siRNA for 48 h. The upper trace of each group displays representative blots of the respective proteins. The trace of each group displays representative blots of the respective proteins. (D) Densitometric results are expressed as a fold increase. (E) Protein expression levels were measured by western blot analysis. The following groups were assessed: i) Control, normal DMEM; ii) TBHP; iii) TBHP + GYY4137, CHs were pre-treated with $100 \mu \mathrm{M}$ GYY4137; iv) TBHP + GRP-78 siRNA, CHs were transfected with 150 nM GRP-78 siRNA for $48 \mathrm{~h}$; and v) TBHP + CQ, CHs were treated with autophagic inhibitor CQ. The upper trace of each group displays representative blots of the respective proteins. (F) Densitometric results are expressed as a fold increase. (G) Protein expression levels were measured via western blotting. The following groups were assessed: i) Control, normal DMEM; ii) TBHP; iii) TBHP + GYY4137, CHs were pre-treated with $100 \mu \mathrm{M}$ GYY4137; and iv) TBHP + GRP-78 siRNA, CHs were transfected with $150 \mathrm{nM}$ GRP-78 siRNA for $48 \mathrm{~h}$. (H) Densitometric results are expressed as a fold increase. ${ }^{*} \mathrm{P}<0.05 \mathrm{vs}$. control; ${ }^{~} \mathrm{P}<0.05$ vs. TBHP. TBHP, tert-Butyl hydroperoxide; $\mathrm{CH}$, chondrocyte; GRP78, 78-kDa glucose-regulated protein; siRNA, small interfering RNA; CQ, chloroquine.

the protective effects of $\mathrm{H}_{2} \mathrm{~S}$ on the $\mathrm{CHs}$ under conditions of oxidative stress.

\section{Discussion}

OA, which leads to joint disorders, is a common disease that primarily affects elderly patients. For the past 20 years, intra-articular treatment options for the management of OA have been limited to strategies that only provide symptomatic relief and are associated with serious adverse effects $(25,26)$.
Therefore, improving the therapeutic strategies is important to improve the efficacy of $\mathrm{OA}$ therapy. $\mathrm{CHs}$ are the unique cell type in the cartilage, which are implicated in multiple stresses, including oxidative stress, inflammation and ER stress. ER stress induces $\mathrm{CH}$ apoptosis, which is associated with the pathogenesis of OA (27). GRP78 has been reported to serve as a critical mediator of ER stress, as demonstrated by decreased mTOR induction (28-33). GRP78 was selected as a novel biomarker, as its level associated with cell process, cell integrity failure, and cell proliferation. In the present study, the suppression of 

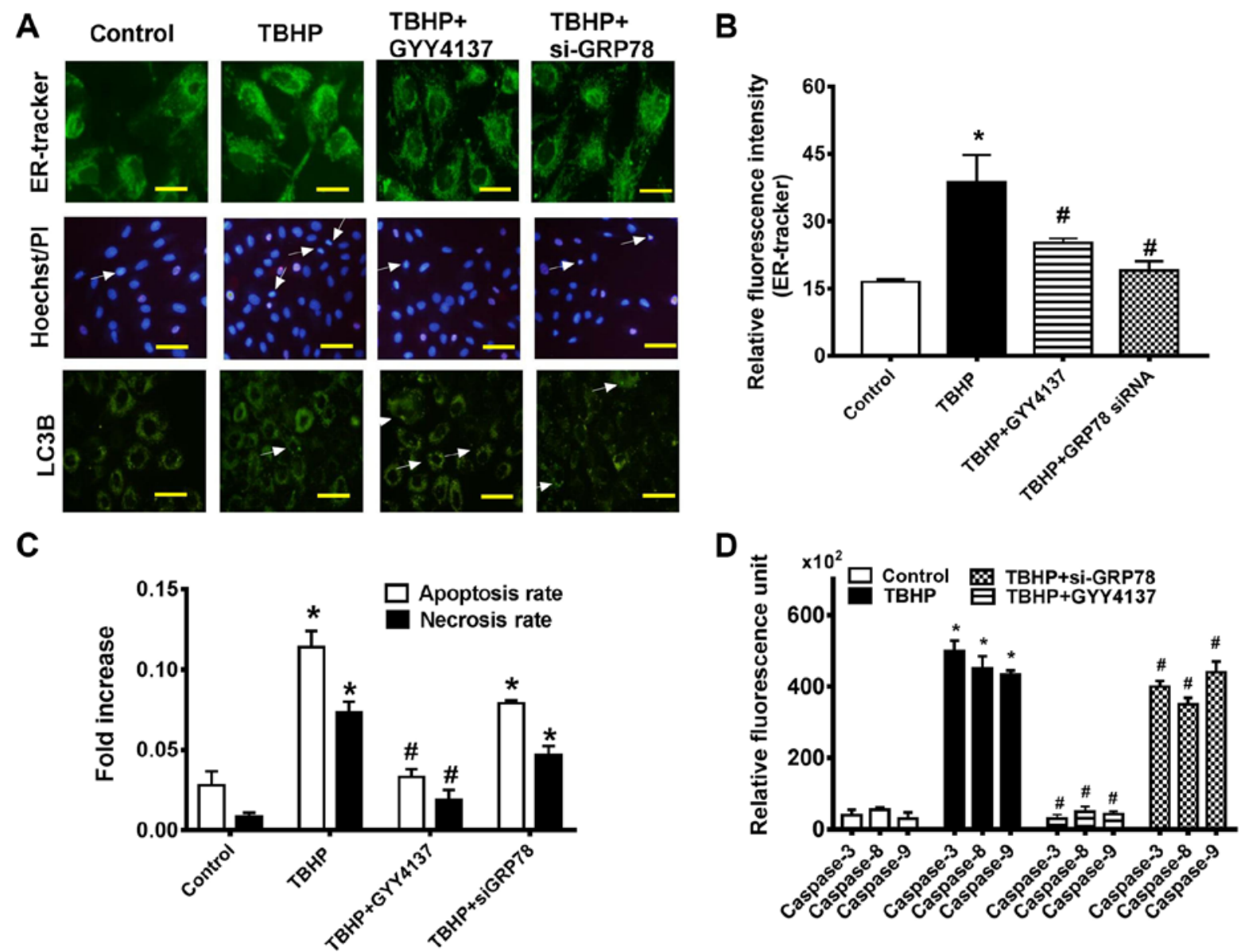

Figure 5. GYY4137 attenuates ER stress via the GRP78/mTOR signaling pathway. (A) Upper panel images: Cells were stained with ER-tracker (green). Fluorescence was observed using a non-confocal fluorescence microscope (magnification, x400). Middle panel images: The effect of GYY4137 and si-GRP78 on the number of apoptotic CHs was assessed by performing Hoechst/PI staining (magnification, x200). Lower panel images: The punctuate dots of a green fluorescent-tagged LC3B protein in CHs following treatment with or without GYY4137 (magnification, x200). (B) Analysis of relative fluorescence intensity in CHs. (C) Quantification of Hoechst-positive cells (as a percentage of total counted cells) and PI-positive cells from 10 fields of view in 5 different areas. (D) The activity levels of caspase- $3,-8$ and -9 in $\mathrm{CHs}$ by relative activity assay. Data are presented as the means $\pm \mathrm{SEM}$. ${ }^{*} \mathrm{P}<0.05 \mathrm{vs}$. control, ${ }^{\#} \mathrm{P}<0.05 \mathrm{vs}$. TBHP $(\mathrm{n}=3)$. ER, endoplasmic reticulum; si-, small interfering RNA; GRP78, 78-kDa glucose-regulated protein; $\mathrm{CH}$, chondrocytes; LC3B, microtubule associated protein 1 light chain $3 \alpha$.

GRP78 was associated with increased mTOR levels (Fig. 4E), which is consistent with its role in cell autophagy (33).

In recent years, $\mathrm{H}_{2} \mathrm{~S}$ has been recognized as a target in cardiovascular diseases, including diabetic cardiomyopathy, pulmonary arterial hypertension and atherosclerosis, but it is relatively novel in $\mathrm{CHs}$. GYY4137, a $\mathrm{H}_{2} \mathrm{~S}$ donor, releases $\mathrm{H}_{2} \mathrm{~S}$ via hydrolysis both in vitro and in vivo, and exhibits prolonged biological activities, including anti-inflammatory, anti-autophagy, anti-apoptotic effects (18-21). The present study aimed to evaluate the protective effects of $\mathrm{H}_{2} \mathrm{~S}$ against OA-induced CH ER stress, as well as the possible effects of $\mathrm{H}_{2} \mathrm{~S}$ on oxidative stress. $\mathrm{H}_{2} \mathrm{~S}$ is a useful antioxidant due to its radical scavenging abilities. Previous in vitro and in vivo studies have demonstrated that exogenous $\mathrm{H}_{2} \mathrm{~S}$ can alleviate cell apoptosis in humans and animals. Both oxidative stress and ER stress have been associated with $\mathrm{CH}$ apoptosis in OA. However, whether $\mathrm{H}_{2} \mathrm{~S}$ can protect $\mathrm{CHs}$ via suppressing ER stress-induced apoptosis is not yet completely understood.

The present study investigated whether $\mathrm{H}_{2} \mathrm{~S}$ prevented apoptosis by inducing oxidative stress in rat $\mathrm{CHs}$, and also explored the possible underlying mechanisms. TBHP was used as an oxidative stress inducer instead of $\mathrm{H}_{2} \mathrm{O}_{2}$ due to its improved stability and longer lasting effects. TBHP has been widely applied in the investigation of the mechanisms underlying OA (31).

In the present study, it was hypothesized that exogenous $\mathrm{H}_{2} \mathrm{~S}$ could protect cartilage against TBHP-induced oxidative stress. The present study relied on the novel finding that GYY4137 displays physicochemical properties that may be related to the inhibition of ER stress-induced apoptosis $(14,17,29)$ via the downregulation of the expression levels of CHOP, GRP78 and ATF6.

Previous studies have suggested another important role for the intrinsic or mitochondrial signaling pathway in transducing a wide spectrum of death signals that originate both inside and outside the cells $(4,15)$. The stimulation of these pathways is mediated by the translocation of death-promoting proteins from the mitochondria to the cytoplasm, which initiates apoptosis via the activation of a class of cysteine proteases (caspases) (16,23). In the present study, GYY4137 treatment markedly decreased the activity of caspase- 3 and decreased the expression levels of p-mTOR (Figs. 3F and 4E) following TBHP stimulation. The results suggested that treatment with exogenous $\mathrm{H}_{2} \mathrm{~S}$ protected against caspase family activation and decreased TBHP-induced $\mathrm{CH}$ apoptosis. Moreover, the results 
indicated that $\mathrm{H}_{2} \mathrm{~S}$ inhibited TBHP-induced ER stress via the apoptotic pathway (Fig. 5A and D). Exogenous $\mathrm{H}_{2} \mathrm{~S}$ treatment and si-GRP78 transfection protected the $\mathrm{CHs}$ against TBHP-induced ER stress compared with the TBHP-cultured group, as measured by ER-tracker and the activity levels of caspase- $3,-8$ and -9 (Fig. 5A).

Autophagy plays as a prominent role in improving cell survival. Previous studies have suggested that the interplay between autophagy and apoptosis is complex (32). LC3 is initially synthesized in its unprocessed form, as evidenced by conversion of LC3I to LC3II. LC3II is localized in the autophagosome membrane and serves as a reliable protein marker of autophagy (34). The results of the present study suggested that the GYY4137-mediated cytoprotective effects against TBHP-induced oxidative stress may be due to its activation of autophagy via the GRP78/mTOR signaling pathway. The results indicated a protective role of GRP78 in CHs. Moreover, si-GRP78 decreased mTOR phosphorylation, but successfully inhibited autophagy, leading to increased apoptosis and cell injury. GRP78 knockdown augmented apoptosis compared with the control group, but suppressed apoptosis compared with the TBHP group (Fig. 5C), suggesting the involvement of GRP78/mTOR-dependent autophagy in cellular-protection. However, the role of autophagy in OA is not yet completely understood, as it is a repair mechanism that is activated by mild stress and with increasing stress levels, apoptosis begins to occur.

A previous study demonstrated that miR-495 targeted GRP78 and activated mTOR signaling to inhibit autophagy in multidrug resistant gastric cancer (GC) cells (33), whereas the present study demonstrated opposite results. First, the inconsistency between the results of the present study and the aforementioned previous study suggested that GC cells and $\mathrm{CHs}$ may be different cell types. Moreover, the inconsistency indicated that there may be an upstream microRNA modulating GRP78 at the post-transcriptional level. In addition to activating mTOR and inhibiting $\mathrm{ROS}, \mathrm{H}_{2} \mathrm{~S}$ may be implicated in preventing apoptosis or inducing autophagy via modulating microRNAs, which should be investigated in future studies. The past quarter-century has witnessed a tremendous expansion in the knowledge of $\mathrm{H}_{2} \mathrm{~S}$ that is associated with anti-apoptotic and anti-oxidative mechanisms. It is worth noting that the development of $\mathrm{H}_{2} \mathrm{~S}$-releasing drugs rarely translates into preclinical success. Thus, it would be important to not only identify the downstream signals of $\mathrm{H}_{2} \mathrm{~S}$, but also production of $\mathrm{H}_{2} \mathrm{~S}$ in various types of cells under normal and disease conditions.

\section{Acknowledgements}

The authors would like to thank Dr Bo Yu from Key Laboratory of Myocardial Ischemia, Ministry of Education, Harbin Medical University (Harbin, China) who was more than generous with his expertise and precious time. His vigorous academic observation enlightens us not only in this thesis but also in our future study.

\section{Funding}

The present study was supported by grants from National Natural Science Foundation of China (no. 81901853) to FY. This study was also supported by the Key Project of Harbin Municipal Science and Technology Bureau (no. 2016RAXYJ068) and dean foundation of the Fourth Affiliated Hospital of Harbin Medical University to DY. The present study was also supported by grants from National Natural Science Foundation of China (no. 81900313) to LY. The funders had no role in the study design, data collection and analysis, decision to publish, or preparation of the manuscript.

\section{Availability of data and materials}

The authors declare that the materials described in the manuscript, including all relevant raw data, will be freely available to any scientist wishing to use them for non-commercial purposes, without breaching participant confidentiality.

\section{Authors' contributions}

DY made substantive contributions to the conception, design of the study and critical revision. JW and FY performed experiments and contributed to the design, data analysis, revision of the original and final manuscript. XZ made contributions to the initial draft of the article and performed experiments. GC, JZ and LY made substantial contributions to the data analysis and interpretation, and revision of the article. All authors read and approved the final manuscript.

\section{Ethics approval and consent to participate}

Ethics approval was obtained from 4th Affiliated Hospital of Harbin Medical University (Harbin, China). Animal experiments were performed according to the Guide for the Care and Use of Laboratory Animals of Harbin Medical University (Harbin, China). All animal experiments were carried out according to animal welfare standards and were approved by the Ethical Committee for Animal Experiments of the Fourth Affiliated Hospital, Harbin, China.

\section{Patient consent for publication}

Not applicable.

\section{Competing interests}

The authors declare that they have no competing interests.

\section{References}

1. Xie J, Lin JT, Wei M, Teng Y, He Q, Yang G and Yang X: Sustained Akt signaling in articular chondrocytes causes osteoarthritis via oxidative stress-induced senescence in mice. Bone Res 7: 23, 2019.

2. Rahmati M, Nalesso G, Mobasheri A and Mozafara M: Aging and osteoarthritis: Central role of the extracellular matrix. Ageing Res Rev 40: 20-30, 2017.

3. Hui W, Young DA, Rowan AD, Xu X, Cawston TE and Proctor CJ: Oxidative changes and signalling pathways are pivotal in initiating age-related changes in articular cartilage. Ann Rheum Dis 75: 449-458, 2016.

4. Wu L and Liu Z: The molecular mechanisms of preventing apoptosis of cartilage chondrocyte to target osteoarthritis. Future Med Chem 9: 537-540, 2017.

5. Li XR, Li J, Ren Q and Sun S: The molecular mechanism of treating osteoarthritis with dipsacus saponins by inhibiting chondrocyte apoptosis. Exp Ther Med 14: 4527-4532, 2017. 
6. Mardones R, Jofre CM, Tobar L and Minguell JJ: Mesenchymal stem cell therapy in the treatment of hip osteoarthritis. J Hip Preserv Surg 4: 159-163, 2017.

7. Hasegawa A, Yonezawa T, Taniguchi N, Otabe K, Akasaki Y, Matsukawa T, Saito Masahiko, Neo M, Marmorstein LY and Lotz MK: Role of fibulin 3 in aging-related joint changes and osteoarthritis pathogenesis in human and mouse knee cartilage. Arthritis Rheumatol 69: 576-585, 2017.

8. da Costa BR, Reichenbach S, Keller N, Nartey L, Wandel S, Juni P and Trelle S: Effectiveness of non-steroidal anti-inflammatory drugs for the treatment of pain in knee and hip osteoarthritis: A network meta-analysis. Lancet 390: e21-e33, 2017.

9. Skrepnik N, Spitzer A, Altman R, Hoekstra J, Stewart J and Toselli R: Assessing the impact of a novel smartphone application compared with standard follow-up on mobility of patients with knee osteoarthritis following treatment with hylan G-F 20: A randomized controlled trial. JMIR MHealth UHealth 5: e64, 2017.

10. Walsh DA: Osteoarthritis: Nerve ablation-a new treatment for OA pain? Nat Rev Rheumatol 13: 393-394, 2017.

11. Gu Y, Chen J, Meng Z, Yao J, Ge W, Chen K, Cheng S, Fu J, Peng L and Zhao YZ: Diazoxide prevents H2O2-induced chondrocyte apoptosis and cartilage degeneration in a rat model of osteoarthritis by reducing endoplasmic reticulum stress. Biomed Pharmacother 95: 1886-1894, 2017.

12. Takada K, Hirose J, Yamabe S, Uehara Y and Mizuta $H$ : Endoplasmic reticulum stress mediates nitric oxide-induced chondrocyte apoptosis. Biomed Rep 1: 315-319, 2013.

13. Couasnay G, Bon N, Devignes CS, Sourice S, Bianchi A, Veziers J, Weiss P, Elefteriou F, Provot S, Guicheux J, et al: $\mathrm{PiT1} / \mathrm{Slc} 20 \mathrm{a} 1$ is required for endoplasmic reticulum homeostasis, chondrocyte survival, and skeletal development. J Bone Miner Res 34: 387-398, 2019.

14. Liu L, Zhang Y, Wang Y,Peng W, Zhang N and Ye Y: Progesterone inhibited endoplasmic reticulum stress associated apoptosis induced by interleukin-1 $\beta$ via the GRP78/PERK/CHOP pathway in BeWo cells. J Obstet Gynaecol Res 44: 463-473, 2018.

15. Ye W, Zhu S, Liao C, Xiao J, Wu Q, Lin Z and Chen J: Advanced oxidation protein products induce apoptosis of human chondrocyte through reactive oxygen species-mediated mitochondrial dysfunction and endoplasmic reticulum stress pathways. Fundam Clin Pharmacol 31: 64-74, 2017.

16. González QM, Blondel A, Sagredo A, Hetz C, Chevet E and Pedeux R: When endoplasmic reticulum proteostasis meets the DNA damage response. Trends Cell Biol 30: 881-891, 2020

17. Yang F, Yu X, Li T, Wu J, Zhao Y, Liu J, Sun A, Dong S, Wu J, Zhong X, et al: Exogenous $\mathrm{H} 2 \mathrm{~S}$ regulates endoplasmic reticulum-mitochondria cross-talk to inhibit apoptotic pathways in STZ-induced type I diabetes. Am J Physiol Endocrinol Metab 312: E190-E203, 2017

18. Whiteman M, Haigh R, Tarr JM, Gooding KM, Shore AC and Winyard PG: Detection of hydrogen sulfide in plasma and knee-joint synovial fluid from rheumatoid arthritis patients: Relation to clinical and laboratory measures of inflammation. Ann N Y Acad Sci 1203: 146-150, 2010.

19. Hou X, Yuan Y, Sheng Y, Yuan B, Wang Y, Zheng J, Liu CF, Zhang $\mathrm{X}$ and Hu LF: GYY4137, an $\mathrm{H}_{2} \mathrm{~S}$ slow-releasing donor, prevents nitrative stress and $\alpha$-synuclein nitration in an MPTP mouse model of Parkinson's disease. Front Pharmacol 8: 741, 2017.

20. John A, Kundu S, Pushpakumar S, Fordham M, Weber G, Mukhopadhyay M and Sen U: GYY4137, a hydrogen sulfide donor modulates miR194-dependent collagen realignment in diabetic kidney. Sci Rep 7: 10924, 2017.
21. Drapala A,KoszelewskiD, Tomasova L, Ostaszewski R, Grman M, Ondrias K and Ufnal M: Parenteral Na2S, a fast-releasing H2S donor, but not GYY4137, a slow-releasing $\mathrm{H}_{2} \mathrm{~S}$ donor, lowers blood pressure in rats. Acta Biochim Pol 64: 561-566, 2017.

22. Feng K, Ge Y, Chen Z, Li X, Liu Z, Li X, Li H, Tang T, Yang F and Wang X: Curcumin Inhibits the PERK-eIF2 $\alpha$-CHOP pathway through promoting SIRT1 expression in oxidative stress-induced rat chondrocytes and ameliorates osteoarthritis progression in a rat model. Oxid Med Cell Longev 2019: 8574386, 2019

23. Zhang G, Wang X, Bi X, Li C, Deng Y, Al-Hashimi AA, Luo X, Gillette TG, Austin RC, Wang Y and Wang ZV: GRP78 (glucose-regulated protein of $78 \mathrm{kDa}$ ) promotes cardiomyocyte growth through activation of GATA4 (GATA-binding protein 4). Hypertension 73: 390-398, 2019.

24. Kumari P, Verma SK and Mobin SM: A facile two-photon fluorescent probe: An endoplasmic reticulum tracker monitoring ER stress and vesicular transport to lysosomes. Chem Commun (Camb) 55: 294-297, 2019.

25. Allen KD, Golightly YM and White DK: Gaps in appropriate use of treatment strategies in osteoarthritis. Best Pract Res Clin Rheumatol 31: 746-759, 2017.

26. Meng $\mathrm{Z}$ and Huang R: Topical treatment of degenerative knee osteoarthritis. Am J Med Sci 355: 6-12, 2018

27. Cao J, Zhang Y, Wang T and Li B: Endoplasmic reticulum stress is involved in baicalin protection on chondrocytes from patients with osteoarthritis. Dose Response 16: 1559325818810636, 2018.

28. Ojha R, Leli NM, Onorati A, Piao S, Verginadis II, Tameire F, Rebecca VW, Chude CI, Murugan S, Fennelly C, et al: ER translocation of the MAPK pathway drives therapy resistance in BRAF-mutant melanoma. Cancer Discov 9: 396-415, 2019.

29. Liu Y, Wang X, Zhen Z, Yu Y, Qiu Y and Xiang W: GRP78 regulates milk biosynthesis and the proliferation of bovinemammaryepithelial cells through the mTOR signaling pathway. Cell Mol Biol Lett 24: 57, 2019.

30. Thon M, Hosoi T, Yoshii M and Ozawa K: Leptin induced GRP78 expression through the PI3K-mTOR pathway in neuronal cells. Sci Rep 4: 7096, 2014

31. Feng C, He K, Zhang C, Su S, Li B, Li Y, Duan CY, Chen S, Chen R, Liu Y, et al: JNK contributes to the tumorigenic potential of human cholangiocarcinoma cells through the mTOR pathway regulated GRP78 induction. PLoS One 9: e90388, 2014.

32. Tang Q, Zheng G, Feng Z, Chen Y, Lou Y, Wang C, Zhang X, Zhang Y, Xu H, Shang P and Liu H: Trehalose ameliorates oxidative stress-mediated mitochondrial dysfunction and ER stress via selective autophagy stimulation and autophagic flux restoration in osteoarthritis development. Cell Death Dis 8: e3081, 2017.

33. Chen S, Wu J, Jiao K, Wu Q, Ma J, Chen D, Kang J, Zhao G, Shi Y, Fan D and Zhao G: MicroRNA-495-3p inhibits multidrug resistance by modulating autophagy through GRP78/mTOR axis in gastric cancer. Cell Death Dis 9: 1070, 2018.

34. Galluzzi L and Green DR: Autophagy-independent functions of the autophagy machinery. Cell 177: 1682-1699, 2019.

This work is licensed under a Creative Common Attribution-NonCommercial-NoDerivatives 4.0 International (CC BY-NC-ND 4.0) License. 